\title{
Late Relapse
}

National Cancer Institute

\section{Source}

National Cancer Institute. Late Relapse. NCI Thesaurus. Code C122607.

Clinical and/or laboratory evidence of late reemergence of a disorder after a period of remission. 